Marquette University

e-Publications@Marquette

2-24-2010

NMR Dynamics Investigation of Ligand-Induced Changes of Main and Side-Chain Arginine N-H's in Human Phosphomevalonate Kinase

Andrew L. Olson

Marquette University

Sheng Cai

Marquette University, sheng.cai@marquette.edu

Timothy J. Herdendorf

University of Missouri - Kansas City

Henry M. Miziorko

University of Missouri - Kansas City

Daniel S. Sem

Marquette University, daniel.sem@marquette.edu

Accepted version. Journal of the American Chemical Society, Vol. 132, No. 7 (February 24, 2010):

2102-2103. DOI. (C) 2010 American Chemical Society. Used with permission. 


\title{
NMR Dynamics Investigation of Ligand-Induced Changes of Main and Side-Chain Arginine N-H's in Human Phosphomevalonate Kinase
}

\author{
Andrew L. Olson \\ Chemical Proteomics Facility at Marquette, Department of \\ Chemistry, Marquette University, \\ Milwaukee, WI \\ Sheng Cai
}

Chemical Proteomics Facility at Marquette, Department of

Chemistry, Marquette University,

Milwaukee, WI

Timothy J. Herdendorf

Division of Molecular Biology and Biochemistry,

School of Biological Sciences,

University of Missouri-Kansas City,

Kansas City, MO

Henry M. Miziorko

Division of Molecular Biology and Biochemistry,

School of Biological Sciences, University of Missouri-Kansas City, Kansas City, MO 
NOT THE PUBLISHED VERSION; this is the author's final, peer-reviewed manuscript. The published version may be accessed by following the link in the citation at the bottom of the page.

\author{
Daniel S. Sem \\ Chemical Proteomics Facility at Marquette, Department of \\ Chemistry, Marquette University, \\ Milwaukee, WI
}

\title{
Abstract
}

\section{Arginine sidechain rigidification}
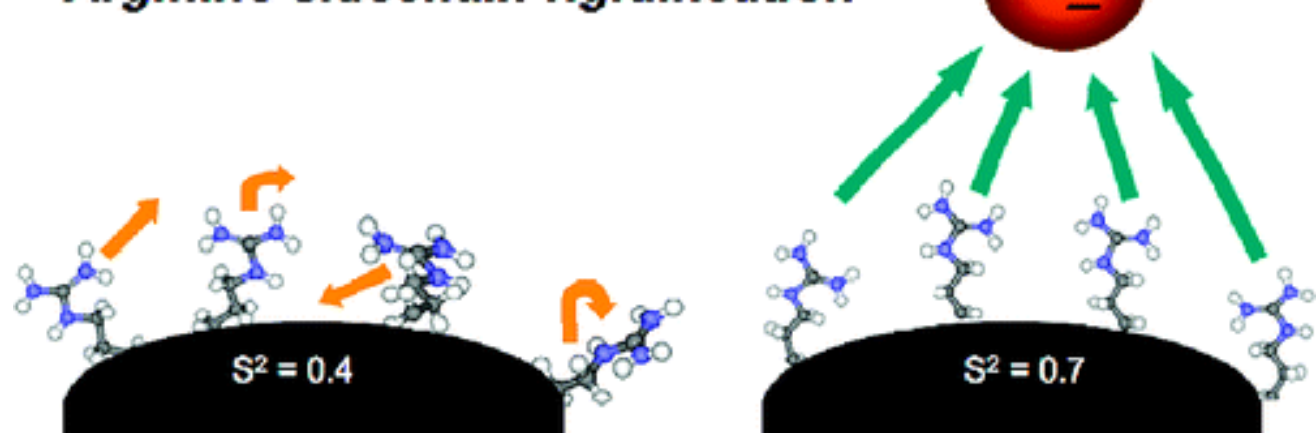

Phosphomevalonate kinase (PMK) catalyzes phosphoryl transfer from adenosine triphosphate (ATP) to mevalonate 5-phosphate (M5P) on the pathway for synthesizing cholesterol and other isoprenoids. To permit this reaction, its substrates must be brought proximal, which would result in a significant and repulsive buildup of negative charge. To facilitate this difficult task, PMK contains 17 arginines and eight lysines. However, the way in which this charge neutralization and binding is achieved, from a structural and dynamics perspective, is not known. More broadly, the role of arginine sidechain dynamics in binding of charged substrates has not been experimentally defined for any protein to date. Herein we report a characterization of changes to the dynamical state of the arginine side chains in PMK due to binding of its highly charged substrates, ATP and M5P. These studies were facilitated by the use of arginine-selective labeling to eliminate spectral overlap. Model-free analysis indicated that while substrate binding has little effect on the arginine backbone dynamics, binding of either substrate leads to significant rigidification of the arginine side chains throughout the protein, even those that are $>8 \AA$ from the binding site. Such a global rigidification of arginine side chains is unprecedented and suggests that there are long-range electrostatic interactions of sufficient strength to restrict the motion of arginine side chains on the picosecond-to-nanosecond time scale. It will be interesting to see whether such effects are general for arginine residues in proteins that bind highly charged substrates, once additional studies of arginine side-chain dynamics are reported. 
Studies of the dynamical properties of proteins using NMR spectroscopy is an emerging field that has largely been limited to backbone $\mathrm{N}-\mathrm{H}$ or side-chain methyl motions. It is often but not always true that changes to the dynamic state of the backbone reflect changes in the side chains. The NMR techniques for quantifying fasttime-scale motion involve measuring the longitudinal $\left(R_{1}\right)$ and transverse $\left(R_{2}\right)$ relaxation rates as well as the heteronuclear nuclear Overhauser effect (NOE) for each amino acid backbone $\mathrm{N}-\mathrm{H}$ (or sidechain $\mathrm{C}-\mathrm{H}$ ) bond vector in the protein. $1-3,10$ These values can then be used to calculate the generalized order parameter $\left(\mathrm{S}^{2}\right)$, which is a measure of protein flexibility.

We have recently reported on the ligand-induced structural and dynamical changes of human phosphomevalonate kinase (PMK). ${ }^{4} \mathrm{PMK}$ is the fifth enzyme in the mevalonate pathway in humans and is involved in steroid biosynthesis. 5,6 PMK catalyzes phosphoryl transfer from adenosine triphosphate (ATP) to mevalonate 5-phosphate (M5P) to form adenosine diphosphate (ADP) and mevalonate 5-diphosphate. To permit phosphoryl transfer, the substrates are brought close together, resulting in a significant and repulsive buildup of negative charge. To facilitate this difficult task, PMK contains 17 arginines (Figure 1) and eight lysines, many of them in the active site to help neutralize the negative charge on the phosphates. The most important arginines, on the basis of site-directed mutagenesis studies, are R18, $R 48, R 73, R 84, R 110, R 111$, and $R 141.7,8$ Here we describe the use of NMR dynamics methods to characterize changes in the mobility of arginine side chains upon ligand binding. NMR dynamics methods, which have recently been applied to arginine side chains, $10,11,13$ allow study of the role that arginine side chains play in ligand binding and catalysis. Our studies provide surprising insights into ligand effects on arginine side chains that are remote from the active site, perhaps due to long-range Coulombic attractions. 


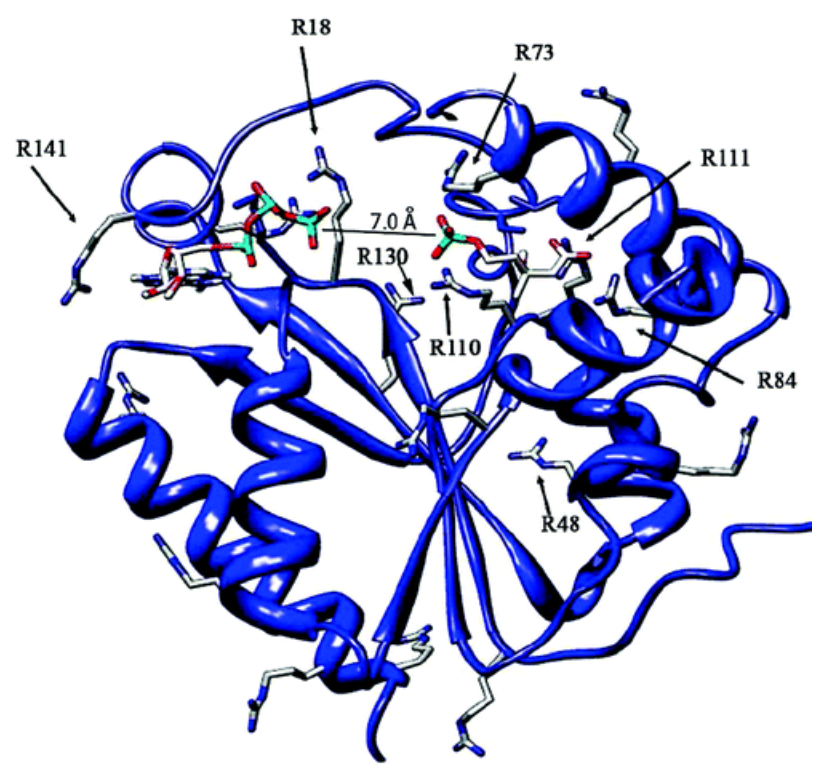

Figure 1. Ternary complex of human PMK, with M5P and ATP docked independently into the apo crystal structure (PDB entry $3 \mathrm{CH} 4) .9$ After the substrates were docked, the structure was optimized using molecular dynamics (50 ps at $300 \mathrm{~K}$ ). All of the arginines are shown, and those known to be important for substrate binding or catalysis are labeled. The interphosphate distance is $7 \AA$, so phosphate transfer requires additional domain movement.

Since PMK is a rather large protein ( $24 \mathrm{kDa})$, in terms of NMR studies, samples were prepared with only arginines having the ${ }^{15} \mathrm{~N}$ label (Figure $\underline{2} \mathrm{~A}$ ). This allowed us to make sure that every arginine backbone residue was considered, without concern for signal overlap, along with the $\varepsilon-\mathrm{N}-\mathrm{H}^{\prime} \mathrm{s}$ of the side chain. Other arginine side-chain $\mathrm{N}-\mathrm{H}^{\prime} \mathrm{s}$, though labeled, exchange too rapidly to be observed. Because PMK has significant resonance overlap in the backbone $\mathrm{N}-\mathrm{H}$ region and also because of significant relaxation that precluded side-chain assignments via TOCSY experiments, only half of the arginine residues could be assigned. The assigned arginines are labeled accordingly on the spectra, and the rest of the arginine backbone $\mathrm{N}-\mathrm{H}^{\prime} \mathrm{s}$ are labeled $\mathrm{R}-\mathrm{A}$ through R-I. The $\varepsilon-\mathrm{N}-\mathrm{H}^{\prime} \mathrm{s}$ of the arginine side chains are shown at the top of Figure $\underline{2} \mathrm{~A}$ and expanded in Figure $\underline{2} \mathrm{~B}$. As these side-chain $\mathrm{N}-\mathrm{H}$ 's could not be assigned to their respective backbone $\mathrm{N}-\mathrm{H}$ 's, they have been labeled SC-1 through SC-13; furthermore, four of the 17 residues are apparently exchange-broadened. 
NOT THE PUBLISHED VERSION; this is the author's final, peer-reviewed manuscript. The published version may be accessed by following the link in the citation at the bottom of the page.

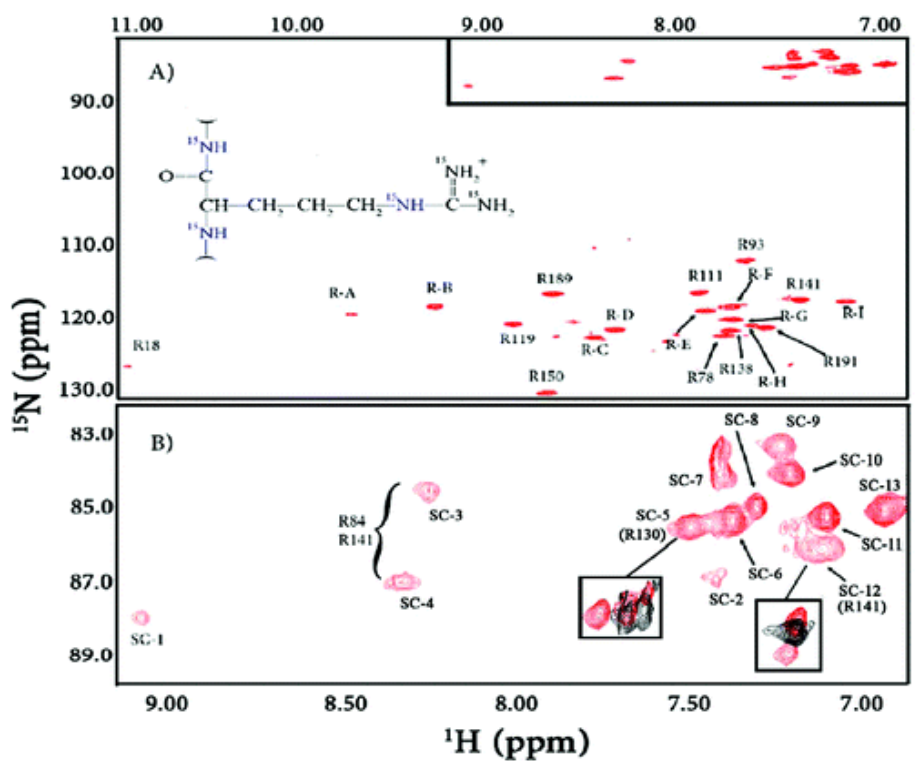

Figure 2. (A) ${ }^{1} \mathrm{H}-{ }^{15} \mathrm{~N}$ HSQC spectrum of ${ }^{15} \mathrm{~N}$-Arg-PMK, with backbone residues labeled. (B) Expansion of the arginine side-chain region, with assignment labels. Overlaid expansions in boxes show data for mutation-based assignments (mutated PMK in black). Spectra of a $500 \mu \mathrm{M}$ PMK solution ( $20 \mathrm{mM}$ potassium phosphate, $5 \mathrm{mM}$ dithiothreitol, $10 \% \mathrm{D}_{2} \mathrm{O}, 10 \%$ glycerol- $d_{6}$ ) were recorded at $298 \mathrm{~K}$ using a $600 \mathrm{MHz}$ Varian NMR system.

To monitor arginine involvement in the binding of PMK's substrates, chemical-shift titration experiments were performed (Figure S1 in the Supporting Information). Increasing amounts of M5P and MgADP were added to PMK, and the arginine backbone and side chains were monitored using ${ }^{1} \mathrm{H}-{ }^{15} \mathrm{~N}$ HSQC spectra. The majority of the arginine $\mathrm{N}-\mathrm{H}^{\prime} \mathrm{s}$ showed very small chemical shift changes, indicating little change in chemical environment for residues labeled SC- 2 and SC-5-13. For residues labeled SC-1, -2 , and -4 , the chemical shifts were exchange-broadened, indicating some change in environment and motion due to binding of M5P (Figure S1C). This experiment was duplicated using MgADP (Figure S1D), monitoring chemical shift changes as before. Only SC-1, -2 , and -4 showed large chemical shift changes, suggesting that they are involved in binding.

Dynamics experiments were performed as before, where we showed that substrate-induced domain movement occurs in PMK. ${ }^{4}$ The longitudinal relaxation rates $\left(R_{1}\right)$ were generally the same for the backbone and side chains for each particular complex and agreed with previous results 4 (Figure S2 and Table S1). The transverse relaxation rates $\left(\mathrm{R}_{2}\right)$ of the backbone $\mathrm{N}-\mathrm{H}^{\prime} \mathrm{s}$ also correlated with previous 
studies. $\underline{4}$ The side chains, however, showed a decrease in transverse relaxation rates relative to the backbone. This decrease in $R_{2}$ values for the apo-PMK arginine side chains was significant (half relative to backbone). The decrease in $\mathrm{R}_{2}$ was small for the M5P/PMK complex, with the other two complexes showing an intermediate decrease. The ${ }^{1} \mathrm{H}-{ }^{15} \mathrm{~N}$ NOE values for the side chains varied from residue to residue, but this variability was consistent between complexes.

As in previous studies, $\stackrel{4}{\text { a }}$ model-free analysis $\underline{2}$ was used to generate the generalized order parameter, $S^{2}$. The $S^{2}$ value is a measure of rigidity (where $S^{2}=1.0$ indicates complete rigidity and $S^{2}$ $=0.0$ complete fluidity) on the nanosecond-to-picosecond time scale for the bond of interest, $\underline{1,2}$ in our case the arginine backbone and sidechain $\mathrm{N}-\mathrm{H}$ bond vectors. Model-free analysis can also differentiate between global and local motions. In our previous work on PMK, we showed that domain movement (global motion) was affected by ligand binding, with little attenuation of local motions. In the side-chain studies presented herein, it was observed that ligand binding causes the arginine $\varepsilon-\mathrm{N}-\mathrm{H}^{\prime} \mathrm{s}$ to increase $\mathrm{S}^{2}$ on average from 0.47 in apo-PMK to $0.64-0.75$ in the binary and ternary complexes (Figure $\underline{3}$ ). This leads to the conclusion that ligand binding causes arginine side chains to rigidify throughout the protein, even distal to the binding site, perhaps as a result of long-range Coulombic interactions with the substrates. The $\mathrm{S}^{2}$ values for the arginine backbones corresponded to our previous results ${ }^{4}$ on fully ${ }^{15} \mathrm{~N}$ labeled PMK, where we reported changes in $\mathrm{T}_{\mathrm{c}}$ due to domain motion, analogous to those in adenylate kinase (Table S1); the differences are attributed to different buffer conditions. Surprisingly, the backbone dynamics changes were minimal, in stark contrast to the side-chain effects. 


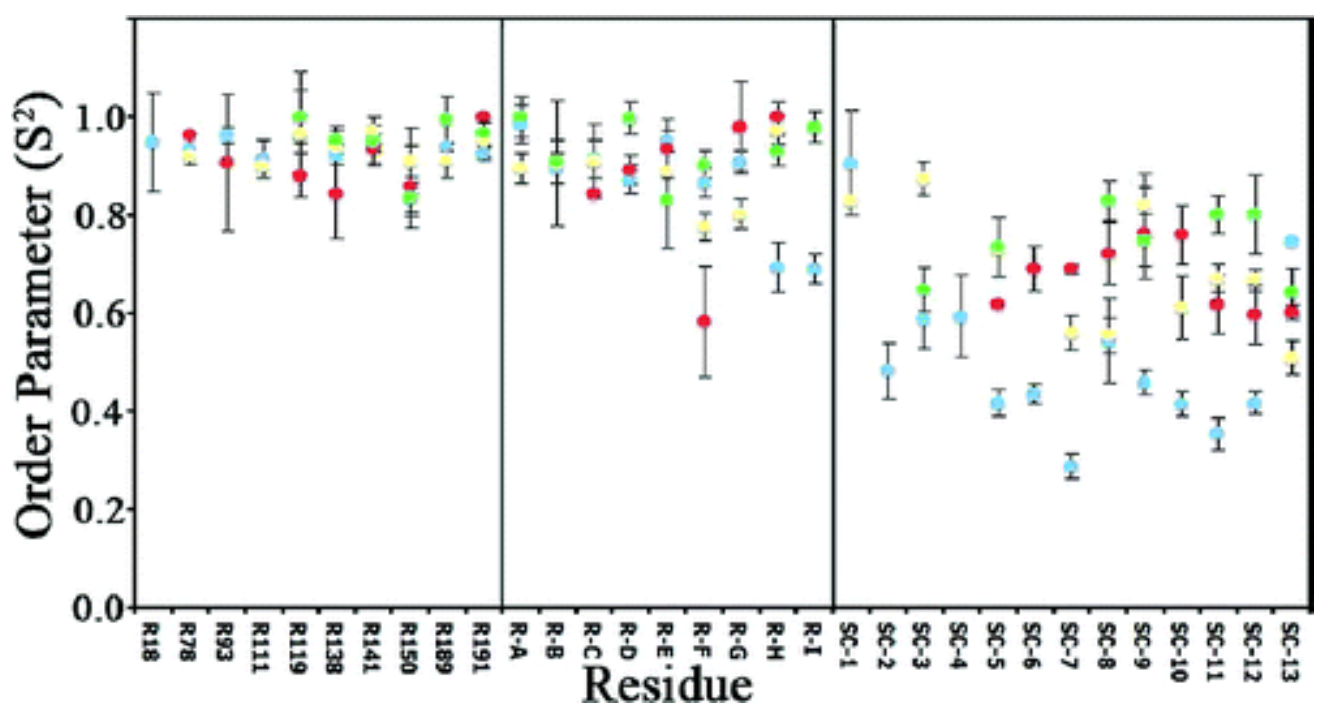

Figure 3. Generalized order parameters ( $\mathrm{S}^{2}$ values) for all assigned and unassigned arginine backbone and side-chain $\varepsilon-\mathrm{N}-\mathrm{H}^{\prime} \mathrm{s}$, where blue refers to apo-PMK, red to the M5P/PMK complex, yellow to the MgADP/PMK complex, and green to the M5P/MgADP/PMK ternary complex. Using site-directed mutagenesis, we have tentatively assigned SC-2 and -3 as R84 and R111, with SC-5 likely being assigned as R130 and SC-12 probably being R141. Nearly all of the arginine side-chain residues undergo a significant increase in $\mathrm{S}^{2}$ (i.e., rigidification) upon binding to either ligand. While Arg84 and Arg111 are close to charged atoms on the substrate, Arg130 and Arg141 are $>10 \AA$ from charged atoms.

It has been noted in studies of side-chain methyl groups that changes in the dynamic state of side chains may not be well-reflected in changes observed in the backbone.12,14 We also have observed quite large differences between the backbone and side-chain responses to substrate binding. Whether such dramatic differences between sidechain and backbone dynamics changes are common in arginines (vs aliphatic side chains) and/or in interactions with highly charged substrates (multiple phosphate groups) cannot be concluded until more arginine side-chain dynamics studies on other proteins are reported.

While it is possible that substrate binding simply causes a global rigidification of side chains in $\mathrm{PMK}$, this seems unlikely. We propose that side chains rigidify because of Coulombic attraction from the highly charged substrate(s); in support of this hypothesis, the ADPinduced $\mathrm{S}^{2}$ effect is abolished in high salt (Figure S8). This must be a long-range effect, since only four arginines are within $4 \AA$ and only six arginines are within $8 \AA$, even though $\sim 10$ arginine side chains rigidify (Figure $\underline{3}$ ). 
In summary, we have shown that substrate binding to PMK causes arginine side chains to undergo a transition from a flexible to a rigid state. The magnitude and global nature of this effect is significant. Besides providing mechanistic insights, such studies facilitate docking by identifying flexible side chains.

\section{Acknowledgment}

This research was supported by funding from the American Heart Association (05303072) to D.S.S. and the NIH (DK53766) to H.M.M. and by NIH-NSF Instrumentation Grants (S 10 RR019012 and CHE-0521323) to the Chemical Proteomics Facility at Marquette. The authors also thank Garrett Mohr for expressing the PMK mutants and Raj Rathore for stimulating discussions.

\section{Supporting Information}

Detailed procedures and additional NMR spectra. This material is available free of charge via the Internet at http://pubs.acs.org.

\section{References}

1 Mandel, A. M., Akke, M. and Palmer, A. G. J. Mol. Biol. 1995, 246, 144

${ }^{2}$ Clore, G. M., Szabo, A., Bax, A., Kay, L. E., Driscoll, P. C. and Gronenborn, A. M. J. Am. Chem. Soc. 1990, 112, 4989

36ay, L. E., Torchia, D. A. and Bax, A. Biochemistry 1989, 28, 8972

4 Olson, A. L., Yao, H., Herdendorf, T. J., Miziorko, H. M., Hannongbua, S., Saparpakorn, P., Cai, S. and Sem, D. S. Proteins 2009, 75, 127

5 Hellig, H. and Popjak, G. J. Lipid Res. 1961, 235

${ }^{6}$ Eyzaguirre, J., Valdebenito, D. and Cardemil, E. Arch. Biochem. Biophys. $2006,454,189$

7Herdendorf, T. J. and Miziorko, H. M. Biochemistry 2006, 45, 3235

8Herdendorf, T. J. and Miziorko, H. M. Biochemistry 2007, 46, 11780

${ }^{9}$ Chang, Q., Yan, X., Gu, S., Liu, J. and Liang, D. Proteins 2008, 73, 254

${ }^{10}$ Buck, M., Boyd, J., Redfield, C., MacKenzie, D. A., Jeenes, D. J., Archer, D. B. and Dobson, C. M. Biochemistry 1995, 34, 4041

11Cai, M., Gong, Y., Wen, L. and Krishnamoorthi, R. Biochemistry 2002, 41, 9572

$12 \mathrm{Hsu}, \mathrm{S}$. T., Cabrita, L. D., Fucini, P., Christodoulou, J. and Dobson, C. M. J. Am. Chem. Soc. 2009, 131, 8366

13 Trbovic, N., Cho, J.-H., Abel, R., Friesner, R. A., Rance, M. and Palmer, A. G. J. Am. Chem. Soc. 2009, 21, 615 
NOT THE PUBLISHED VERSION; this is the author's final, peer-reviewed manuscript. The published version may be accessed by following the link in the citation at the bottom of the page.

${ }^{14}$ Igumenova, T. I., Frederick, K. and Wand, A. J. Chem. Rev. 2006, 106, 1672

Journal of the American Chemical Society, Vol 132, No. 7 (February 24, 2010): pg. 2102-2103. DOI. This article is (C) American Chemical Society and permission has been granted for this version to appear in e-Publications@Marquette. American Chemical Society does not grant permission for this article to be further copied/distributed or hosted elsewhere without the express permission from American Chemical Society. 